\title{
Rol y condiciones estratégicas del médico dentro del Sistema de Gestión de Seguridad y Salud Ocupacional
}

\section{Conditions and Strategic Role of the Doctor within the Occupational Health and Safety Management System}

\section{Óscar Eduardo Vera-Romero 1-8, Virgilio Efraín Failoc-Rojas', Franklin Miguel Vera-Romero 1,5}

1. Universidad Nacional Pedro Ruiz Gallo - UNPRG, Lambayeque, Perú

2. Universidad Nacional de Trujillo, La Libertad, Perú

3. Órgano de apoyo al Comité Editor de la Revista del Cuerpo Médico del Hospital Nacional Almanzor Aguinaga Asenjo, Chiclayo, Perú

4. Red Peruana de Divulgadores Científicos de Lambayeque, Chiclayo, Perú

5. TMental Group: Scientific Research in the Health Sciences, Chiclayo, Peru

6. Clínica Cayetano Heredia, Huancayo, Perú

7. Clínica Internacional de Especialidades Médicas, Huancayo, Perú

8. Policlínico Médicos Sin Fronteras, Huancayo, Perú

Recibido: 13-10-14

Aceptado: 12-01-15

\section{Correspondencia}

Óscar Eduardo Vera Romero

Jr. Los Manzanos $N^{\circ} 105$

El Tambo - Huancayo, Perú.

Correo electrónico: leoedu $1 @$ hotmail.com; tmentalgroup@outlook.com

Resumen

El impacto negativo de los accidentes laborales es tanto sanitario como económico. Se calcula que cerca de 2,3 millones de muertes al año se deben por trabajo. Existen factores de riesgo, como exposición a riesgos físicos, químicos, biológicos, psicosociales y ergonómicos ocasionando desequilibrio de salud y causar accidentes, enfermedades. Para proteger la salud en estos campos existe la Medicina del Trabajo. El Objetivo de este estudio es describir el rol y condiciones estratégicas del médico dentro del sistema de gestión de seguridad y salud ocupacional. Materiales y Métodos: Revisión bibliográfica en BIREME, Scielo Perú, España, Colombia y publicaciones de la International Labour Organización (ILO). Conclusión: El Rol Estratégico del Médico dentro del Sistema de Gestión de Seguridad y Salud Ocupacional presenta los enfoques: humano, médico-legal, económico y social; con responsabilidad directa en la implementación de políticas y programas de salud ocupacional, basado en los códigos de ética nacional e internacional.

Med Segur Trab (Internet) 2015; 61 (238) 34-44

Palabras clave: Salud Laboral, Rol del Médico, Medicina del Trabajo, Agotamiento profesional (Fuente: DeCS, BIREME)

Abstract

The negative impact of occupational accidents is both health and economic. It is estimated that close to 2.3 million deaths a year are due for work. There are risk factors such as exposure to physical risks, chemical, biological, psychosocial, and ergonomic causing imbalance in health and cause accidents, diseases. To protect health in these fields there is a Occupational Medicine. The objective of this study is to describe 
the role and strategic conditions of the doctor within the occupational health and safety management system. Materials and methods: review of the literature in BIREME, Scielo Peru, Spain, Colombia, and publications of the International Labour Organization (ILO). Conclusion: The strategic role of the doctor within the Occupational Health and Safety Management System presents the approaches: human, medical-legal, economic and social; with direct responsibility for the implementation of policies and programs of occupational health, based on national and international ethical codes.

Med Segur Trab (Internet) 2015; 61 (238) 34-44

Key Words: Occupational Health, Occupational Medicine, Physician's Role, Burnout Professional (Source: MeSH, PUBMED). 


\section{INTRODUCCIÓN}

Los accidentes laborales son causas de enorme impacto sanitario y económico para los trabajadores, las empresas y la sociedad en general. Este impacto negativo es reflejado en las muertes, incapacidades y sufrimiento personal de los trabajadores, así como el ausentismo laboral y la pérdida de productividad. Según cifras de la OIT (Organización Internacional del Trabajo), se calcula que cada año en todo el mundo se producen cerca de 2,3 millones de muertes debido a enfermedades provocadas por trabajo, mientras que el número anual total de casos de enfermedades profesionales no mortales se calcula en 160 millones ${ }^{1}$. En el Perú ocurren 18 muertes al año en accidentes de trabajo de un total de cien mil empleados y estas ocurren principalmente en los sectores construcción, industria y minería ${ }^{2}$.

Las condiciones de seguridad son esenciales para la realización de cualquier actividad laboral, sin embargo, es común encontrarse con situaciones peligrosas, denominados «Factores de Riesgo Laboral", como la exposición a riesgos físicos, químicos, biológicos, psicosociales y ergonómicos ${ }^{3}$. Estos factores pueden acarrear al desequilibrio de salud, y causar accidentes, enfermedades profesionales y otras relacionadas con el ambiente laboral ${ }^{4,5}$. En el Perú, se desconoce los diferentes riesgos ocupacionales a la cual está expuesta la población trabajadora y no se cuenta con información estadística sobre enfermedades y accidentes de trabajo ${ }^{5}$; en las cuales las exigencias legales para el control de los riesgos ocupacionales no son tenidas en cuenta por negligencia o desconocimiento, causando así accidentes y enfermedades en los trabajadores.

Es por eso que la Medicina del Trabajo, rama de la Medicina Humana, se dedica a proteger la salud amenazada por el trabajo, con acciones sobre el trabajador e indirectamente sobre el puesto de labor y el ambiente. Tiene carácter integral: asistencial, preventivo-promocional, rehabilitador y, en última instancia, curativo. Pero, el carácter patognomónico de la especialidad es su ejercicio ligado a los programas de seguridad e higiene de la empresa ${ }^{6}$. Su ejercicio sobre el trabajador es esencial y armónico, debiendo ser ejecutada por el equipo de Salud Ocupacional (SO), incluyendo sus familias y su ambiente. Los componentes esenciales radican en la evaluación periódica de la salud del trabajador, la evaluación médica preempleo, la vigilancia médica, el diagnóstico y tratamiento de las enfermedades derivadas del trabajo, los accidentes laborales y extralaborales, la rehabilitación, la educación de los trabajadores hacia la identificación y control de los riesgos potenciales para la salud, la implementación de programas para el uso apropiado de equipos de protección personal, evaluaciones toxicológicas, evaluación e inspección de los lugares de trabajo, el mantenimiento de registros médicos confidenciales, programas de inmunización contra enfermedades prevenibles, evaluación bioestadística y epidemiológica, interpretación médica y participación en regulaciones en salud y seguridad, preparación para desastres y asistencia en la rehabilitación de los trabajadores con problemas de adicción ${ }^{7,8}$.

Por esa razón, SO es un pilar elemental en el progreso de un país, sus funciones están dirigidas a la promoción y protección de la salud de los trabajadores y la prevención de accidentes de trabajo y enfermedades ocupacionales ocasionadas por las condiciones de trabajo y riesgos ocupacionales en las diversas actividades económicas. Por lo tanto, el rol del Medico dentro del Sistema de Gestión de Seguridad y Salud Ocupacional, es muy importante, debido a que su desempeño se basa en la Medicina del Trabajo, permitiendo el mantenimiento y mejoramiento de sus condiciones de salud. Siendo esta, la base para una gestión activa de la Seguridad y la salud Ocupacional.

\section{METODOLOGÍA}

El presente trabajo de investigación es Observacional-Descriptivo de Diseño Transversal. Partiendo del marco conceptual descrito por el Manual de Salud Ocupacional del Ministerio de Salud del Perú conjuntamente con La Organización Panamericana de 
Salud (MINSA Perú/OPS) (7) y la revisión bibliográfica en BIREME, Scielo Perú, España, Colombia y publicaciones de la International Labour Organization (ILO); para estandarizar criterios sobre el rol de médico ocupacional.

El presente estudio tiene como objetivo describir el rol y condiciones estratégicas del médico dentro del sistema de gestión de seguridad y salud ocupacional.

Aspectos éticos: El estudio de revisión y análisis se realizó de acuerdo a las Pautas Éticas Internacionales. Además, se tuvieron en cuenta las siguientes consideraciones: Prevalece los principios de Beneficencia y de Justicia. Por lo tanto, no implicaron riesgo alguno para seres humanos.

\section{REVISIÓN DE TEMA}

\section{Situación Actual del Medico Ocupacional en el Perú}

Hasta hace unas décadas atrás, las funciones de los médicos eran fundamentalmente clínico-asistenciales, y en parte orientadas hacia los problemas de salud no necesariamente relacionados con el trabajo ${ }^{1,2,6}$. Sin embargo, surgen dilemas éticos sobre la protección del empleo y la salud; frente a esa situación, la Organización Mundial de la Salud (OMS) y la Organización Internacional del Trabajo (OIT) en el año de 1950, formulan la práctica de la Salud Ocupacional, siendo actualizados en 1995, por el Comité Conjunto de Salud Ocupacional OIT/OMS, siendo sus objetivos primordiales: a) La promoción y mantenimiento del más alto grado de bienestar físico, mental y social de los trabajadores en todas las ocupaciones, b) La prevención de daños a la salud causados por sus condiciones de trabajo, c) La ubicación y mantenimiento de trabajadores en un ambiente de trabajo adaptado a sus capacidades fisiológicas y psicológicas, d) La adaptación del trabajo al hombre y de cada hombre a su tarea, y e) El desarrollo de organizaciones y culturas de trabajo basados en la salud y seguridad en el trabajo, promoviendo ambientes sociales positivos y una operación que permita la productividad de los procesos.

En el Perú, la Medicina Ocupacional se inicia formalmente el 5 de Agosto de 1940 cuando se crea por Decreto Supremo el Departamento Nacional de Higiene Industrial. En 1957 con la reestructuración del Ministerio de Salud Pública y Asistencia Social cambió su nombre a Instituto de Salud Ocupacional. Sus primeras acciones estuvieron avocadas al análisis situacional de la salud ocupacional en el país, encontrándose que era la actividad minera la que tenía los más altos índice de morbi-mortalidad. Por ello los primeros esfuerzos preventivos fueron dirigidos hacia dicha actividad industrial. Durante el siglo XXVI, aparece el Decreto Supremo N. ${ }^{\circ}$ 009-2005-TR, donde se reglamentó sobre Seguridad y Salud en el Trabajo. Luego, la Ley de Seguridad y Salud en el Trabajo ( ${ }^{\circ}$ 29783), publicada como Decreto Supremo N. ${ }^{\circ}$ 005-2012-TRel 20 de agosto de 2011, estableciéndose la obligación empresarial de contar con un Sistema de Gestión en Seguridad y Salud en el Trabajo (SG-SST) en todas las empresas públicas o privadas. Finalmente, el 11 de Julio del 2014, el Congreso de la República publicó la Ley No 30222 que modifica la Ley 29783 sobre Seguridad y Salud en el Trabajo, permitiendo facilitar su implementación, manteniendo el nivel efectivo de protección de la salud y seguridad y reduciendo los costos para las unidades productivas y los incentivos a la informalidad.

Por lo expuesto, en el Perú, se trata de una especialidad médica nueva, reconocida por el Colegio Médico bajo dos modalidades, una como Médico Especialista en Medicina del Trabajo y otra como Médico Especialista en Medicina Ocupacional y del Medio Ambiente. Actualmente se extiende los estudios de especialización en la modalidad de Diplomados en diversa universidades tanto públicas como privadas. Sin embargo, hace algunos años, la Universidad Peruana Cayetano Heredia estuvo extendiendo por estudios de posgrado, el título de Especialista en Medicina del Trabajo ${ }^{5,7}$. 


\section{Médico de Medicina del Trabajo o Salud Ocupacional}

Debe ser médico especializado en medicina del trabajo, tiene que participar en la evaluación ocupacional de la empresa para conocer de primera mano sus instalaciones y los riesgos presentes o potenciales. El médico ocupacionista deviene en el pilar del equipo, aunque por sí solo es insuficiente para desarrollar los programas de salud ocupacional.

Las actividades que realiza el médico en medicina ocupacional van a depender de las circunstancias donde las practique, las que pueden estar circunscritas al área pública o privada. Sin embargo, las medidas más importantes han sido relacionadas al trabajo multidisciplinario con epidemiólogos, toxicólogos, higienistas industriales e ingenieros de seguridad, dado por el emergente desarrollo del enfoque multidisciplinario de la Salud Ocupacional ${ }^{5,6}$.

Figura 1. Funciones de los médicos del trabajo e historia natural de la enfermedad

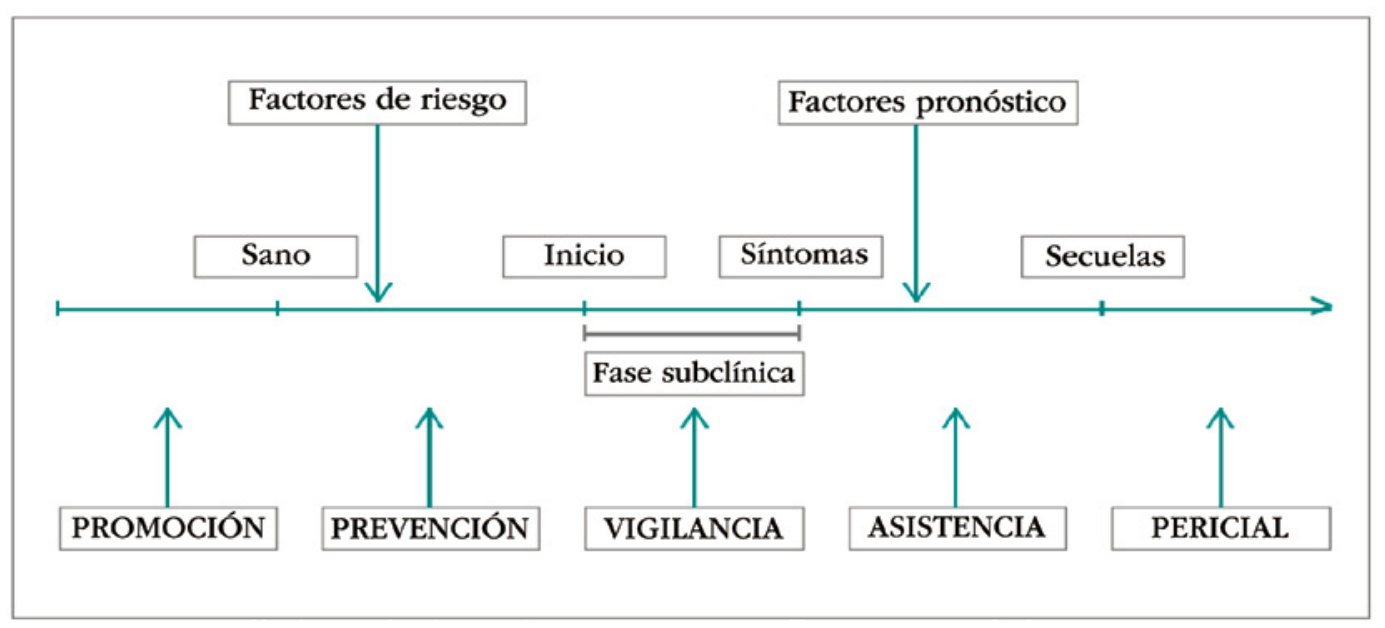

Tomado: Las competencias profesionales de los médicos del trabajo. Publicado por el Instituto de Salud Carlos II, Universitat Pompeu Fabra, Cyclops. Barcelona 2003

Además, no se están limitando a la realización de evaluaciones periódicas y provisión de servicios, sino que se buscan atender la salud del trabajador y su capacidad para trabajar en su puesto o ambiente de trabajo, con la intención de protegerlo y hacerlo más productivo en base a su capacidades físicas y mentales y dirigido a sus necesidades humanas y sociales. Este enfoque incluye la atención preventiva, la promoción de la salud, los servicios asistenciales tradicionales curativos, la rehabilitación a través de los primeros auxilios y la compensación económica cuando corresponda, así como las estrategias para la recuperación y reinserción laboral ${ }^{6-8}$.

\section{Cualidades que debe tener: Dentro de las capacidades y habilidades a poseer, debe destacar}

a) Habilidad para administrar un programa médico ocupacional que incluya a todos los trabajadores; b) Poseer cualidades y conocimientos que le permitan asesorar a la dirección de la empresa y a los trabajadores en el campo médico; c) Conocer de los informes médicos por accidentes de trabajo; d) recomendar acciones sobre incapacidades laborales; e) Apoyar al departamento legal de la empresa, de presentarse algún litigio laboral sobre aspectos médicos; f) Supervisar las actividades de sus asistentes médicos, de enfermería y de técnicos ocupacionales; g) Organizar el sitio de trabajo técnica y normativamente para realizar las evaluaciones médicas por riesgos; $y, h$ ) Preparar informes y estadística para la gerencia, para los trabajadores y para auditorías internas o legales, según corresponda. 
Por lo tanto, estas nuevas funciones conllevan también a que el profesional médico, posea nuevas competencias y a ejercer una medicina orientada al trabajo en sus muy diversos ámbitos; principalmente en los servicios de prevención de las empresas, propios y externos; en diversos servicios de la administración (Unidades de Salud Laboral, Unidades de Valoración de Daños); sindicatos, instituciones académicas y de investigación, y quizás en la inspección de trabajo como ocurre en otros países ${ }^{1,5}$.

Tabla 1. Cuadro Resumen del Rol Estratégico del Médico dentro del Proceso de un Sistema de Gestión de Seguridad y Salud Ocupacional

\begin{tabular}{|c|c|c|c|}
\hline España & Perú & Funciones & Competencias \\
\hline PROMOCIÓN & PROMOCIÓN & $\begin{array}{l}\text { Conjunto de actividades } \\
\text { cuyo objetivo es mejorar } \\
\text { el nivel de salud de los } \\
\text { trabajadores mediante } \\
\text { intervenciones destinadas } \\
\text { a capacitarlos para } \\
\text { incrementar el control } \\
\text { sobre su salud y } \\
\text { mejorarla8, tanto frente a } \\
\text { los riesgos laborales como } \\
\text { extra-laborales. }\end{array}$ & $\begin{array}{l}\text { 1. Fomentar la cultura preventiva en } \\
\text { la empresa y conductas, hábitos, } \\
\text { consumos y estilos de vida saludables. } \\
\text { 2. Fomentar la participación activa de los } \\
\text { trabajadores como protagonistas de } \\
\text { su salud. } \\
\text { 3. Desarrollar programas sanitarios } \\
\text { frente a patologías prevalentes en la } \\
\text { comunidad. } \\
\text { 4. Fomentar la creación de entornos } \\
\text { saludables en la empresa. } \\
\text { 5. Participar en los programas de salud } \\
\text { organizados por las instituciones } \\
\text { sanitarias. }\end{array}$ \\
\hline
\end{tabular}

PREVENCIÓN DIFUSIÓN Conjunto de actividades cuyo objetivo es reducir o eliminar riesgos laborales mediante intervenciones colectivas o personales.
1. Asesorar a la empresa, a los trabajadores $\mathrm{y}$ a sus representantes en todos los aspectos de la prevención de riesgos.

2. Proponer medidas preventivas a partir de la evaluación de riesgos.

3. Planificar la prestación de los primeros auxilios en los centros de trabajo.

4. Indicar la adaptación de las condiciones de trabajo a los trabajadores en general y de los susceptibles en particular.

5. Informar y formar sobre los riesgos laborales para la salud y las medidas preventivas necesarias, como inmunoquimioprofilaxis necesaria frente a los riesgos laborales.

6. Participar en la identificación evaluación y prevención de los riesgos medioambientales comunitarios derivados de las actividades de las empresas.

7. Evaluar la efectividad y eficiencia de las medidas preventivas implementadas para eliminar y reducir la exposición a partir de los indicadores de salud.

\begin{tabular}{lll}
\hline FORMACIÓN & DISEÑO DE & Conjunto de procesos \\
& PROGRAMAS Y \\
POLÍTICAS & búsquemático en la de soluciones.
\end{tabular}

1. Identificar las necesidades formativas y fomentar el aprendizaje permanente propio y ajeno.

2. Diseñar, realizar y evaluar actividades de formación.

3. Seleccionar y aplicar las técnicas de comunicación interpersonal.

4. Utilizar las tecnologías de comunicación e información. 


\begin{tabular}{|c|c|c|}
\hline España & Perú & Funciones \\
\hline GESTIÓN & & $\begin{array}{l}\text { Se refiere exclusivamente } \\
\text { al servicio de prevención } \\
\text { planificado y normado } \\
\text { para toda la empresa. }\end{array}$ \\
\hline
\end{tabular}

Competencias

1. Utilizar las técnicas de gestión y organización de recursos y actividades. para toda la empresa.

2. Aplicar criterios de calidad en la gestión del servicio.

3. Gestionar la confidencialidad de la información sanitaria.

4. Trabajar de forma integrada en el equipo multidisciplinar de prevención.

5. Ejercer una medicina del trabajo de acuerdo con criterios éticos.

6. Promover prácticas socialmente responsables en relación a la salud de los trabajadores.

VIGILANCIA EJECUCIÓN Conjunto de actividades cuyo objetivo es la detección precoz de alteraciones de salud, principalmente relacionados con el trabajo, mediante procedimientos de recogida sistemática y análisis de información tanto a nivel individual como colectivo.
1. Diseñar las actividades de vigilancia de la salud adecuadas a cada situación con criterios de validez.

2. Identificar, diagnosticar, Analizar y Valorar los problemas de salud relacionados con el trabajo y su interacción con el trabajo (capacidad laboral).

3. Aplicar técnicas de control Biológico, Químico, Físico, Ergonómico de exposición e interpretar sus resultados.

4. Analizar los distintos registros o fuentes de información sanitaria disponibles.

5. Intercambiar e integrar informaciones de forma bidireccional con el resto del equipo multidisciplinar.

6. Realizar encuestas de salud y llevar a cabo los exámenes de salud e interpretar sus resultados.

7. Identificar la información sanitaria de interés, analizarla con criterios epidemiológicos y manejar indicadores de salud.

8. Comunicar e informar los resultados de la vigilancia de forma asertiva.

9. Promover medidas de adecuación del trabajo al trabajador con un problema de salud. 


\begin{tabular}{cll}
\hline España & \multicolumn{1}{c}{ Perú } & \multicolumn{1}{c}{ Funciones } \\
\hline ASISTENCIA & EVALUACIÓN & Conjunto de actividades \\
& & que tienen como objetivo \\
& el manejo clínico y laboral \\
& de los trabajadores con \\
& un problema de salud, \\
& principalmente aquél \\
& relacionado con las \\
& condiciones de trabajo.
\end{tabular}

Competencias

1. Proporcionar la atención médica necesaria ante emergencias y urgencias.

2. Llevar a cabo una historia clínica y laboral, así como una exploración clínica completas.

3. Evaluar la interacción entre una especial susceptibilidad individual y el trabajo.

4. Evaluar el riesgo derivado de la situación de salud del trabajador para sí mismo o terceros.

5. Realizar el diagnóstico, tratamiento y seguimiento de las enfermedades profesionales, enfermedades relacionadas con el trabajo y enfermedades comunes que en su caso proceda.

6. Interactuar con el sistema público de salud en la prevención, diagnóstico, tratamiento y rehabilitación de los problemas de salud.

7. Evaluar las consecuencias de los posibles tratamientos médicos que puedan limitar la capacidad en el trabajo.

8. Elaborar y promover recomendaciones sobre rehabilitación y reincorporación al trabajo.

INVESTIGACIÓN MONITOREO Conjunto de actividades cuyo objetivo es identificar, cuantificar y valorar las secuelas de los daños a la salud relacionados con el trabajo y su impacto sobre la capacidad para trabajar con el fin de compensar social y económicamente al trabajador afectado.
1. Promover una medicina del trabajo basada en la evidencia.

2. Buscar y analizar la documentación científica existente.

3. Conocer los sistemas nacionales e internacionales de investigación y desarrollo.

4. Formular y gestionar proyectos de investigación.

5. Llevar a cabo investigaciones científicas sobre los problemas de salud relacionados con el trabajo y sus causas.

6. Difundir los resultados y conclusiones de la investigación adecuadamente.

PERICIAL

1. Utilizar adecuadamente los instrumentos de valoración del daño y aplicarlos en el diagnóstico precoz de los problemas de salud relacionados con el trabajo.

2. Valorar el daño corporal tras un problema de salud relacionado con el trabajo.

3. Informar y asesorar adecuadamente al trabajador afectado, a las administraciones y tribunales. 


\section{Condiciones para el Desempeño de las Funciones de los Profesionales Médicos de la Salud Ocupacional}

Siendo el objetivo principal del ejercicio de la salud en el trabajo el preservar y promover la salud de los trabajadores, un medio ambiente de trabajo sano y seguro, proteger la capacidad laboral de los trabajadores y su acceso al empleo. El profesional de la salud ocupacional lo logrará mediante el asesoramiento continuo, honesto y competente a la dirección ejecutiva y a los trabajadores sobre los factores existentes en la empresa, conduciendo al establecimiento de una política de salud y seguridad en el trabajo y a un programa de prevención adaptables al tiempo y las condiciones existentes según la evolución de la empresa ${ }^{9}$. Teniendo como base el conocimiento científico y técnico actualizado, disponible y dentro del marco legal establecido en dicho momento, así como su propio conocimiento de la organización y el ambiente de trabajo, generando oportunas medidas correctivas, de monitoreo y control de los riesgos con su reducción de sus consecuencias en el caso de fracaso. Finalmente, los médicos ocupacionales deben ser conscientes de su rol en relación con la protección de la comunidad y el ambiente, dentro del marco de la salud pública ${ }^{10}$.

Tabla 2. Cuadro sobre las condiciones para el desempeño profesional del Médico dentro del Proceso de un Sistema de Gestión de Seguridad y Salud Ocupacional

\begin{tabular}{|c|c|}
\hline Cualidad & Condiciones para su desempeño \\
\hline integridad & $\begin{array}{l}\text { Debe actuar siempre como cuestión prioritaria en defensa de la salud y seguridad de } \\
\text { los trabajadores. } \\
\text { No se debe obtener ningún tipo de información personal que no sea pertinente para la } \\
\text { protección, mantenimiento y promoción de la salud de los trabajadores en relación con } \\
\text { su trabajo o la salud general de la fuerza de trabajo. }\end{array}$ \\
\hline Competencia & $\begin{array}{l}\text { Debe basar sus juicios en los conocimientos científicos y su competencia técnica, } \\
\text { solicitar asesoramiento a expertos especializados cuando lo estime necesario. }\end{array}$ \\
\hline Imparcialidad & $\begin{array}{l}\text { Debe abstenerse de emitir juicios, dar consejos o realizar actividades que puedan poner } \\
\text { en peligro la confianza en su integridad e imparcialidad. }\end{array}$ \\
\hline $\begin{array}{l}\text { Independencia } \\
\text { Profesional }\end{array}$ & $\begin{array}{l}\text { Debe procurar mantener plena independencia profesional y observar las normas de } \\
\text { confidencialidad en el ejercicio de sus funciones. No deben permitir que sus juicios } \\
\text { y declaraciones se vean influenciados por conflictos de intereses (empleadores, } \\
\text { trabajadores o grupos laborales), sobre el abordaje de los riesgos y las situaciones que } \\
\text { muestren evidencia de peligro para la salud. }\end{array}$ \\
\hline $\begin{array}{l}\text { Equidad y No } \\
\text { discriminación }\end{array}$ & $\begin{array}{l}\text { Todos los trabajadores deben ser tratados de manera equitativa, sin ser objeto de } \\
\text { ninguna discriminación en relación a su condición, convicciones o razón que lo } \\
\text { condujo a consultar al profesional de salud ocupacional. }\end{array}$ \\
\hline Comunicación & $\begin{array}{l}\text { Establecer y mantener canales de comunicación abiertos entre ellos (médicos) y el } \\
\text { funcionario o ejecutivo de la empresa responsable de las decisiones de más alto nivel y } \\
\text { lo representantes de los trabajadores. }\end{array}$ \\
\hline $\begin{array}{l}\text { Clausura ética en } \\
\text { los contratos de } \\
\text { empleo }\end{array}$ & $\begin{array}{l}\text { Los profesionales de la Salud ocupacional deben solicitar la inclusión de una clausula } \\
\text { ética en sus contratos de trabajo, que les permita como especialistas de la Salud } \\
\text { Ocupacional, aplicar estándares, guías y códigos de ética. Así mismo, no deben de } \\
\text { aceptar condiciones de ejercicio que no les permitan desempeñar sus funciones de } \\
\text { acuerdo con las normas y principios de ética profesional. Además, los contrataos de } \\
\text { trabajo deben contener disposiciones sobre aspectos legales, contractuales y éticos del } \\
\text { manejo de conflictos, sobre todo acerca a los registros y de la confidencialidad; como } \\
\text { también que no contengan disposiciones o cláusulas que limiten su independencia } \\
\text { profesional, siendo a veces necesario solicitar asesoramiento jurídico competente. }\end{array}$ \\
\hline Registros & $\begin{array}{l}\text { Se debe mantener registros adecuados con el nivel de confidencialidad óptimo, con el } \\
\text { objetivo de determinar los problemas de salud ocupacional de la empresa. Conteniendo } \\
\text { la información vigilante sobre datos personales, historia de exposición ocupacional } \\
\text { previa, el ambiente de trabajo actual, salud ocupacional del trabajador, resultados } \\
\text { del monitoreo laboral actual y los certificados de aptitud. Siendo necesario que los } \\
\text { trabajadores tengan acceso a dicha información. }\end{array}$ \\
\hline
\end{tabular}




\begin{tabular}{|c|c|}
\hline Cualidad & Condiciones para su desempeño \\
\hline $\begin{array}{l}\text { Confidencialidad } \\
\text { Médica }\end{array}$ & $\begin{array}{l}\text { Los datos obtenidos de la investigación médica continua sobre el estado de la salud } \\
\text { ocupacional de la empresa y los trabajadores deben de guardarse de forma segura bajo } \\
\text { la responsabilidad del profesional encargado de toda la Política de Seguridad y Salud } \\
\text { en el Trabajo, principalmente médico. Dicha información solo podrá utilizarse para } \\
\text { fines de la salud ocupacional. Por lo que el acceso, a las fichas o archivos médicos; así } \\
\text { como su trasmisión, divulgación y utilización se rige por las leyes o normas nacionales } \\
\text { que existan y por los códigos de ética para los profesionales médicos y de la salud. }\end{array}$ \\
\hline $\begin{array}{l}\text { Información } \\
\text { sobre salud } \\
\text { colectiva }\end{array}$ & $\begin{array}{l}\text { Es necesario porque permite realizar informes sobre la salud colectiva de los } \\
\text { trabajadores a la dirección y a los representantes de los trabajadores en la empresa, } \\
\text { o a los comités de salud y seguridad en el trabajo cuando existan; a fin de ayudarles } \\
\text { a cumplir con sus obligaciones de proteger la salud y la seguridad de los grupos de } \\
\text { trabajadores expuestos a riesgos. Se debe notificar los accidentes de trabajo y las } \\
\text { enfermedades ocupacionales a las autoridades competentes de conformidad con las } \\
\text { leyes y las normas nacionales vigentes. }\end{array}$ \\
\hline $\begin{array}{l}\text { Relaciones con } \\
\text { otros profesionales } \\
\text { de la Salud }\end{array}$ & $\begin{array}{l}\text { Los profesionales de la Salud Ocupacional deben colaborar con otros profesionales de } \\
\text { la salud respecto a la protección de la confidencialidad de los datos médicos y de salud } \\
\text { de los trabajadores. } \\
\text { Los médicos ocupacionales pueden solicitar datos o información a los médicos } \\
\text { personales o al personal médico de los hospitales siempre que cuenten para ello con } \\
\text { el consentimiento informado del trabajador y que sea con el propósito de proteger, } \\
\text { mantener o promover su salud. De la misma forma, el médico u otro profesional del } \\
\text { trabajo pueden informar al médico particular o del hospital sobre los datos relativos a } \\
\text { la salud de éste; así como los factores de riesgo, las exposiciones ocupacionales y las } \\
\text { limitaciones en el trabajo que entrañe un riesgo especial para ese trabajador debido a } \\
\text { su estado de salud. }\end{array}$ \\
\hline $\begin{array}{l}\text { Lucha contra los } \\
\text { Abusos }\end{array}$ & $\begin{array}{l}\text { Cuando sea necesario, los médicos ocupacionales deben identificar, evaluar e informar } \\
\text { a las autoridades competentes sobre los procedimientos o prácticas que se estén } \\
\text { aplicando y que a su juicio sean contrarios a los principios éticos establecidos en } \\
\text { la norma legal existente. Este se relaciona con el abuso o el uso inadecuado de la } \\
\text { información de salud ocupacional, la adulteración o retención de hallazgos, la violación } \\
\text { de la confidencialidad médica o la protección inadecuada de los archivos guardados en } \\
\text { forma virtual en computadoras. }\end{array}$ \\
\hline $\begin{array}{l}\text { Relaciones con } \\
\text { los interlocutores }\end{array}$ & $\begin{array}{l}\text { Debe existir una buena relación con todos los miembros de la empresa. Debiendo } \\
\text { sensibilizar a los empleados, los trabajadores y sus representantes respecto a la } \\
\text { necesidad de la plena independencia profesional y al compromiso de proteger la } \\
\text { confidencialidad médica, con el objetivo de respetar la dignidad humana y contribuir a } \\
\text { la aceptación y la eficacia de la práctica de la salud ocupacional. }\end{array}$ \\
\hline $\begin{array}{l}\text { Promoción de la } \\
\text { Ética }\end{array}$ & $\begin{array}{l}\text { El profesional de la salud en el trabajo debe buscar el apoyo y la cooperación de } \\
\text { los empleadores, los trabajadores y sus organizaciones, así como de las autoridades } \\
\text { competentes, para aplicar los más rigurosos estándares éticos en el ejercicio de la salud } \\
\text { ocupacional. }\end{array}$ \\
\hline $\begin{array}{l}\text { Auditoria } \\
\text { profesional }\end{array}$ & $\begin{array}{l}\text { El médico ocupacional debe instituir programas de auditoría profesional de sus propias } \\
\text { actividades para garantizar que los estándares establecidos son los adecuados, que se } \\
\text { están cumpliendo, para que las deficiencias que puedan presentarse sean detectadas y } \\
\text { corregidas, asegurando el mejoramiento continuo del desempeño profesional. }\end{array}$ \\
\hline
\end{tabular}

\section{CONCLUSIÓN}

El Rol Estratégico del Médico dentro del Sistema de Gestión de Seguridad y Salud Ocupacional es de responsabilidad directa en la implementación de políticas y programas de salud ocupacional; basados en el código de ética propuesta por la comisión Internacional de Salud Ocupacional (CISO/ICOH), desglosándose de la siguiente manera:

- Humana: frente a una enfermedad ocupacional o un accidente laboral que afecta al trabajador, también compromete al entorno familiar, de la empresa y a la colectividad. 
- Médico: La práctica de la Salud Ocupacional debe realizarse de acuerdo con los estándares profesionales más altos y los principios éticos más rigurosos. Siendo parte de estas obligaciones la integridad de la conducta profesional, la imparcialidad y la protección de la confidencialidad de los datos sobre la salud y la privacidad de los trabajadores.

- Legal: Sin duda para una empresa es la razón primordial, pues la ley determina como obligatorio mantener instalaciones y condiciones de trabajo que garanticen la salud y seguridad de sus trabajadores. Incluye la protección de la Vida, la Salud y el Respeto por la Dignidad Humana.

- Económica: La razón de ser de los programas de prevención-promoción de la salud no solo tiene carácter humano, moral y social, sino que el plus del control de accidentes y enfermedades laborales para las empresas es la disminución de costos en primas de seguros y en costos directos al mejorar su productividad.

- Social: Por naturaleza, el hombre es un ser social, y prevenir una enfermedad o accidente debe ser compromiso de la empresa con el trabajador y con la sociedad.

\section{REFERENCIAS BIBLIOGRÁFICAS}

1. International Labour Organization. La Prevención De Las Enfermedades Profesionales: Día Mundial de la Seguridad y Salud en el Trabajo 28 de abril de 2013. Safework. 2013. (15 slider). Disponible en: http:// www.ilo.org/wcmsp5/groups/public/---ed_protect/---protrav/---safework/documents/presentation/ wcms_207970.pdf

2. Sánchez Castro CL, Toledo Ríos GZ. Estudio, análisis y evaluación de la siniestralidad laboral en las empresas del sector construcción [Tesis para optar el Título de Ingeniería Industrial]. Lima: Pontificia Universidad Católica Del Perú; 2013.

3. Oficina Internacional del trabajo. Seguridad y salud en el Trabajo. Primera edición. Ginebra; 2009.

4. Ramírez AV. Servicios de Salud Ocupacional. Rev An. Fac. Med. 2012; 73(1):63-69.

5. Ministerio de salud, Dirección General de Salud Ambiental- DIGESA. Manual de salud ocupacional. Lima: 2005.

6. McCunney RJ. A Managers Guide to Occupational Health Services. Environmental Medical Service. Cambridge, Massachusetts, USA: MIT, 2004.

7. Gomero-Cuadra R, Llap-Yesan, C. La Medicina Ocupacional en los últimos tiempos. Rev Med Hered. 2005; 16(4):273-275.

8. Salazar-Gómez CA, Corrales-Hernández JR. Rol del médico especialista en salud ocupacional en la identificación, evaluación y calificación de las enfermedades derivadas del estrés ocupacional. Rev CES Salud Pública. 2012;3(1):104-107.

9. Gastanaga M. Salud Ocupacional: Historia y Retos del Futuro. Rev. med. exp. Salud Publica. 2012; 29 (2):177-178

10. Gomero-Cuadra R, Zevallos-Enriquez C, Llap-Yesan C. Medicina del Trabajo, Medicina Ocupacional y del Medio Ambiente y Salud Ocupacional. Rev Med Hered. 2006; 17(2):105-108. 